\title{
Influence of alloying on the electron momentum density in the $\mathrm{Cu}-\mathrm{Ni}$ system
}

\author{
C. Metz and Th. Tschentscher* \\ European Synchrotron Radiation Facility (ESRF), Boite Postale 220, 38043 Grenoble, France \\ T. Sattler, K. Höppner, and J. R. Schneider \\ Hamburger Synchrotronstrahlungslabor (HASYLAB) at Deutsches Elektronen-Synchrotron (DESY), Notkestrasse 85, \\ 22603 Hamburg, Germany \\ K. Wittmaack \\ GSF-Forschungszentrum für Umwelt und Gesundheit, Institut für Strahlenschutz, 85764 Neuherberg, Germany \\ D. Frischke and F. Bell \\ Sektion Physik der Universität München, Am Coulombwall 1, 85748 Garching, Germany
}

(Received 27 April 1999)

\begin{abstract}
We report on the measurement of the three-dimensional electron momentum density (EMD) of a $22 \mathrm{~nm}$ $\mathrm{Cu} / 22 \mathrm{~nm} \mathrm{Ni}$ sandwich foil and of a $\mathrm{Cu}_{0.50} \mathrm{Ni}_{0.50}$ alloy film with the same thickness, which was obtained from an identical sandwich by interdiffusion. The EMD's were measured by coincident detection of a Compton scattered photon with its recoil electron. The experiments were performed at the High-Energy beamline of the European Synchrotron Radiation Facility. The experimentally observed small change of the EMD due to alloying is reproduced by the Korringa-Kohn-Rostoker coherent-potential approximation scheme [Benedek et al., Phys. Rev. B 32, 7650 (1985)]. [S0163-1829(99)08143-6]
\end{abstract}

\section{INTRODUCTION}

For quite a long time Compton scattering has been used to characterize the electron momentum density (EMD) of valence electrons in solids. ${ }^{1}$ The double-differential cross section describing the energy and angular distribution of inelastically scattered hard $\mathrm{x}$-rays is proportional to the so-called Compton profile (CP), which is defined as a twofold integration over the EMD. This integration results from the lack of information about the momentum distribution of the recoiling electrons. Since integration averages over large volumes in momentum space, detailed information about solid-state effects like the influence of alloying in compound systems might become difficult to obtain. It is therefore desirable to measure the EMD directly by fixing the scattering kinematics: if the momentum of the recoiling electron is measured simultaneously with that of the scattered x-ray photon, the momentum of the electron in its initial state can be reconstructed in a unique way. The corresponding tripledifferential cross section is proportional to the EMD itself. ${ }^{2-5}$ The main difficulty of such a $(\gamma, e \gamma)$ coincidence experiment originates from the strong incoherent elastic scattering of the recoiling electron within the target, which disturbs the determination of the recoil momentum by multiple scattering. Since the mean free path for elastic scattering of electrons with an energy of $50 \mathrm{keV}$ in $\mathrm{Cu}$ is only about $12 \mathrm{~nm},{ }^{6}$ targets as thin as possible are required.

In this paper we will report on the influence of alloying by comparing the EMD of $\mathrm{Cu}_{0.50} \mathrm{Ni}_{0.50}$ with those of its pure constituents. The electronic structure of this transition-metal solid-solution alloy has received extensive attention. The $\mathrm{Cu}-\mathrm{Ni}$ system is completely soluble over the whole concentration range, i.e., there is no miscibility gap yielding single phase disordered fcc alloys. The lattice parameter changes between $\mathrm{Cu}$ and $\mathrm{Ni}$ by $2 \%$ only and the density by $0.3 \%{ }^{7}$ Thus, a change of the electronic structure will not be obscured by geometrical effects. Substantial improvement has been achieved in the theoretical understanding of the electronic structure of disordered alloys using a general multiplescattering formalism. Three principal approximations have been investigated: (i) the virtual-crystal approximation (VCA), where the effective potential of the alloy is the average of that of the pure constituents; (ii) an average $t$-matrix approximation; and (iii) the coherent-potential approximation (CPA), where a self-consistency requirement is introduced to obtain the single-site $t$ matrix. ${ }^{8,9}$ It is this property of the CPA that makes it preferable to apply, especially for alloys of high concentrations. The underlying Green'sfunction technique of the multiple-scattering problem is similar to that of the Korringa-Kohn-Rostoker (KKR) theory. ${ }^{10,11}$ In a series of papers Bansil and co-workers ${ }^{12-17}$ have extensively investigated the $\mathrm{Cu}-\mathrm{Ni}$ system theoretically. In addition, Temmerman, Gyorffy, and Stocks ${ }^{18}$ have used KKR-CPA for a detailed study of the Bloch spectral function in these alloys. On the experimental side poly-crystalline $\mathrm{Cu}_{x} \mathrm{Ni}_{1-x}$ alloys have been investigated by conventional Compton scattering, ${ }^{19}$ and the CP's were compared with those from KKR-CPA calculations. Qualitative agreement was found, though at small momenta the influence of alloying was predicted by theory to be stronger than revealed by experiment. Differences of $\mathrm{Cu}_{x} \mathrm{Ni}_{1-x}$ directional Compton profiles provided by investigations of single crystals $^{20}$ have been compared with relativistic KKR-CPA calculations. Whereas the calculations cited above hold for paramagnetic alloys only, this new computation includes the ferromagnetic behavior of Ni-rich alloys. Especially at momenta below 0.5 
a.u., the experimental anisotropies are not well described by theory.

A method that also measures EMD's is angular correlation of annihilation radiation (ACAR), though, strictly speaking, it measures the electron-positron pair density, i.e., the electron momentum space wave function weighted by the positron wave function within the solid. ${ }^{21}$ Until now only long-slit experiments, i.e., 1D-ACAR (Ref. 22) or investigations with the crossed-slit geometry, ${ }^{23-26}$ have been reported for the $\mathrm{Cu}-\mathrm{Ni}$ system, but no complete 2D-ACAR experiment. The main topic of these early investigations was the reduction of the Fermi surface (FS) extension with increasing $\mathrm{Ni}$ concentrations, especially the decrease of the neck radius at the $L$ point of the Brillouin zone. The change of the FS by alloying in the $\mathrm{Cu}-\mathrm{Ni}$ system is fairly well described by KKR-CPA theory. ${ }^{27,28}$

\section{METHOD}

If a photon with energy $\omega$ and momentum $\mathbf{k}$ is scattered at an electron with band energy $\varepsilon>0$ and momentum $\mathbf{p}$, the final photon energy $\omega^{\prime}$ and momentum $\mathbf{k}^{\prime}$ is connected with the final electron energy $E^{\prime}$ and momentum $\mathbf{p}^{\prime}$ via energy and momentum conservation

$$
\begin{gathered}
\varepsilon(\mathbf{p})=\omega-\omega^{\prime}-E^{\prime}, \\
\mathbf{p}=\mathbf{k}^{\prime}+\mathbf{p}^{\prime}-\mathbf{k} .
\end{gathered}
$$

Thus, if $\mathbf{k}, \mathbf{k}^{\prime}$, and $\mathbf{p}^{\prime}$ are known experimentally, the initial momentum $\mathbf{p}$ can be reconstructed in a unique way. This demands the coincident detection of both the scattered photon $\left(\omega^{\prime}, \mathbf{k}^{\prime}\right)$ and the recoil electron $\left(E^{\prime}, \mathbf{p}^{\prime}\right)$. The coincidence count rate will be proportional to the triple-differential cross section, which can be written within the so-called impulse approximation:

$$
\frac{d^{3} \sigma}{d \omega^{\prime} d \Omega_{\gamma} d \Omega_{e}}=\frac{\omega}{\omega^{\prime}} p^{\prime}\left(\frac{d \sigma}{d \Omega}\right)_{\mathrm{KN}} \rho(\mathbf{p}),
$$

where $(d \sigma / d \Omega)_{\mathrm{KN}}$ is the Klein-Nishina cross section for linearly polarized photons and $\rho(\mathbf{p})$ is the EMD. Since our experiment cannot distinguish between different valence bands, $\rho(\mathbf{p})$ is the total EMD, i.e., summed over all electronic states. The basic idea of the experiment is the following: first, a double layer (sandwich) consisting of equal amounts of pure $\mathrm{Cu}$ and $\mathrm{Ni}$ is investigated, yielding the average EMD $\rho=\left(\rho_{\mathrm{Cu}}+\rho_{\mathrm{Ni}}\right) / 2$. The sample then is heated such that complete interdiffusion takes place. Since the $\mathrm{Cu}-\mathrm{Ni}$ system has no miscibility gap, a homogeneous alloy is formed that again is measured in order to obtain the EMD of the alloy. The influence of alloying is studied by taking the difference of both experiments. Due to the multiplescattering problem, very thin foils have to be used, and therefore interdiffusion occurs within moderate times at not too high temperatures.

\section{EXPERIMENT}

The experiment was performed at the High-Energy X-Ray Scattering beamline ID15A of the European Synchrotron Radiation Facility ESRF. ${ }^{29}$ An asymmetric wiggler with seven

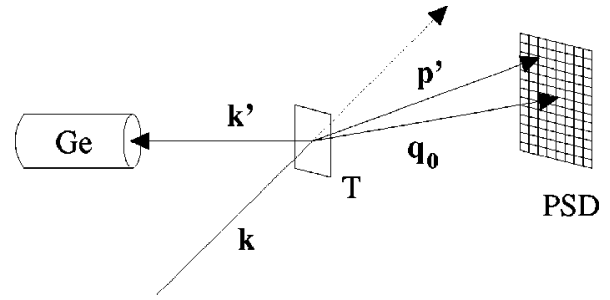

FIG. 1. Experimental setup: Ge, Ge diode; T, target; PSD, position sensitive electron detector.

periods and strong poles of $1.8 \mathrm{~T}$ was used with a critical energy of $44.1 \mathrm{keV}$. The white beam was monochromatized by a (220) bent $\mathrm{Si}$ crystal in Laue geometry. The photon energy was $\omega=146 \mathrm{keV}$ with $\Delta \omega=0.71 \mathrm{keV}$ full width at half maximum (FWHM). The photon beam entered an evacuated target chamber $\left(10^{-3} \mathrm{~Pa}\right)$ with an externally mounted intrinsic Ge-diode (energy resolution $0.4 \mathrm{keV}$ FWHM at $100 \mathrm{keV}$ ) at a scattering angle $\theta=140^{\circ}$. Thus, the scattered photon energy was about $97 \mathrm{keV}$ and the electron recoil energy $49 \mathrm{keV}$. The electrons were measured by a two-dimensional position sensitive detector, which consisted of $16 \times 16$ individual photodiodes (Fig. 1). The center of the array was placed in the direction of the momentum-transfer vector $\mathbf{q}_{0}=\mathbf{k}-\mathbf{k}_{0}^{\prime}$, where $\mathbf{k}_{0}^{\prime}$ is the momentum of photons scattered at electrons at rest. Since both the energy resolution of the photon and electron detector are large compared to the binding energy $\varepsilon$ of the valence electrons, which dominate the EMD, the latter has been neglected in Eq. (2.1a). Detailed Monte Carlo (MC) simulations of the momentum resolution included the correlated scattering due to the cross section of Eq. (2.2), solid angle and energy resolution of the $\mathrm{Ge}$ diode, energy broadening of the primary beam, and extension of the beam spot at the target. The variance vector for the momentum uncertainties in the three Cartesian directions of momentum space obtained by these $\mathrm{MC}$ calculations was $\sigma_{\mathbf{p}}=0.14,0.38,0.19$ a.u. Here $p_{z}$ is parallel to $\mathbf{q}_{0}, p_{x}$ lies in the $\left(\mathbf{k}, \mathbf{k}^{\prime}\right)$ scattering plane, and $p_{y}$ is perpendicular to it. Emission patterns of the recoiling electrons were recorded by the $2 \mathrm{D}$ electron detector with a granularity of about 0.14 a.u. in $p_{x}$ and 0.28 a.u. in $p_{y}$ direction. Thus, the variance in $p_{x}$ and $p_{y}$ direction extended over approximately 1 pixel. The time resolution of the coincidence circuit was about $200 \mathrm{~ns}$, considerably larger than the bunch distance of $3 \mathrm{~ns}$ in the so-called 2/3 fill mode of the ESRF. Time correlation spectra showed very little chance coincidences, which nevertheless were taken into account. The overall coincidence rate due to a primary beam of about $2 \times 10^{11}$ photons/s was about $2 \mathrm{~Hz}$. A total of $1.4 \times 10^{6}$ coincidence events for each experiment was accumulated.

Since self-supporting $\mathrm{Cu}$ or $\mathrm{Ni}$ foils with diameters of 8 $\mathrm{mm}$ and thicknesses of $20 \mathrm{~nm}$ cannot be prepared, we have evaporated $22 \mathrm{~nm} \mathrm{Cu}$ followed by $22 \mathrm{~nm} \mathrm{Ni}$ on a $30 \mathrm{~nm}$ thin $\mathrm{C}$-foil acting as a backing. The backing was made by condensation of evaporated carbon atoms on a thin betaine film that had a fine crystalline-like structure that acted as a replica for the carbon backing and guaranteed a high mechanical stability. Finally, the betaine substrate was dissolved in water, and the carbon film was mounted on a frame. Both $\mathrm{Cu}$ and $\mathrm{Ni}$ were evaporated on the free-standing carbon backing with a rate of about $0.5 \mathrm{~nm} / \mathrm{s}$, and the films condensated at 
room temperature. In order not to lose beam time by the heating procedure, we decided to prepare two sandwich foils simultaneously where the second was heated at about $500^{\circ} \mathrm{C}$ for $2 \mathrm{~h}$ and then furnace cooled. The data of Almazouzi et al. $^{30}$ yield a diffusion coefficient of about $D=2$ $\times 10^{-19} \mathrm{~m}^{2} / \mathrm{s}$ giving for a heating time of $t=7 \times 10^{3} \mathrm{~s}$ a diffusion length $x=2 \sqrt{D t}=80 \mathrm{~nm}$. This is four times the $\mathrm{Cu}$ (or Ni) thickness. Since the diffusion coefficient holds for single crystals, we believe that this diffusion length is a lower limit only. It is well known that especially at rather low temperatures $\left(500^{\circ} \mathrm{C}\right)$ pipe diffusion along grain boundaries or dislocations can be orders of magnitude faster than volume diffusion. ${ }^{31}$ Experimental details of this type of diffusion in the $\mathrm{Cu}-\mathrm{Ni}$ system can be found in Refs. 32-34. Since we expect that our sandwich foils contain quite a lot of defects like grain boundaries and dislocations, diffusion short circuits starting from these defects within the foils will occur and thus shorten the effective diffusion time for complete interdiffusion substantially. The data of Almazouzi et al. ${ }^{30}$ refer to the diffusion of $\mathrm{Ni}$ into a $\mathrm{Cu}$ single crystal. Although the diffusion coefficient of $\mathrm{Cu}$ into a $\mathrm{Ni}$ matrix is about three orders of magnitude smaller than that of Ni into $\mathrm{Cu}$ at $500{ }^{\circ} \mathrm{C}$ (Ref. 35), we expect no void formation due to the Kirkendall effect ${ }^{36}$ at the backing-alloy interface. This is supported by our experience that also after heating the alloy film did not lose its good mechanical contact with the carbon substrate. But it cannot be excluded that vacancy precipitation takes place inside the alloy. The diffusion coefficients cited above are so-called impurity diffusion coefficients in contrast to chemical diffusion coefficients, which hold for the interdiffusion in inhomogeneous binary alloys. ${ }^{36}$ Brunel, Cizeron and Lacombe ${ }^{37}$ measured the chemical diffusion constant over the whole concentration range of the $\mathrm{Cu}-\mathrm{Ni}$ system for temperatures between $700^{\circ} \mathrm{C}$ and $1070{ }^{\circ} \mathrm{C}$. Extrapolating their data to $500^{\circ} \mathrm{C}$ yields diffusion coefficients in the range of $10^{-19} \mathrm{~m}^{2} / \mathrm{s}$ changing by a factor of 5 over the concentration range. These findings agree with ${ }^{63} \mathrm{Ni}$ tracer diffusion in a $\mathrm{Cu}_{0.79} \mathrm{Ni}_{0.21}$ alloy investigated by Butrymowicz, Manning and Read $^{38}$ yielding a diffusion coefficient of the same order of magnitude.

Both foils, the sandwich and the alloy, have been characterized by x-ray fluorescence analysis (XRF), elastic recoil detection analysis (ERDA), and secondary ion mass spectroscopy (SIMS). In XRF the ratio $R=N_{\mathrm{Cu}} / N_{\mathrm{Ni}}$ could be determined where $N_{\mathrm{Cu}}$ and $N_{\mathrm{Ni}}$ are the atomic number densities of both elements. The $K \alpha$ lines of $\mathrm{Cu}$ and $\mathrm{Ni}$, excited by the $146 \mathrm{keV}$ primary photon beam, are well resolved by the $\mathrm{Ge}$ diode (except that the $K \alpha$ line of $\mathrm{Cu}$ is slightly contaminated by the Ni $K \beta$ line, an effect which is easily accounted for). Correcting the line intensities for the ratio of the photoelectric cross sections and the fluorescence yields the ratio $R$ was close to unity within $2 \%$ for both foils, indicating that in fact equal amounts of $\mathrm{Cu}$ and $\mathrm{Ni}$ had been deposited on the backing. Since the foils are extremely thin, the probability that $\mathrm{Cu} K \beta$ radiation can excite Ni $K$ fluorescence is negligible. ERDA reveals the contamination of the targets with low- $Z$ material. In short, $150 \mathrm{MeV} A u$ ions from the Munich heavy-ion accelerator have been directed on the target foils, and the recoil ions ejected from the target have been analyzed by an ionization chamber. ${ }^{39}$ Analysis of the nuclear charge $Z$ of the recoil ions was achieved via the

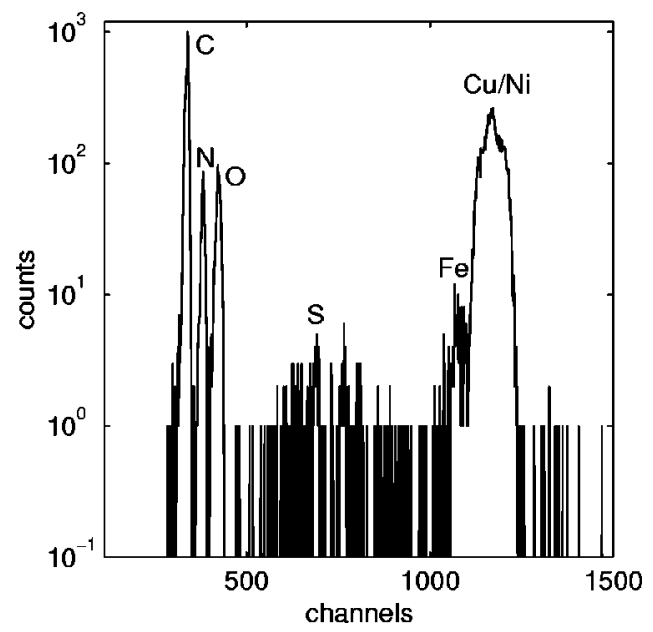

FIG. 2. Elemental composition of the sandwich target from ERDA. Elements are indicated. $\mathrm{Cu}$ and $\mathrm{Ni}$ are not resolved.

specific energy loss $\Delta E$ of the ions within the ionization chamber $\left(\Delta E \sim Z^{2}\right)$. Since the cross section for ejectile emission is the well-known Rutherford cross section, the analysis of low- $Z$ contaminants can be made quantitatively. Figure 2 shows a spectrum of ejectile ions from our foils. In addition to strong peaks from the $\mathrm{C}$ backing and the nonresolved $\mathrm{Cu}$ and $\mathrm{Ni}$ ions, the essential contaminants are oxygen and nitrogen. Quantitative analysis yields concentrations of about 5 at. \% nitrogen and 7 at. \% oxygen, which are slightly reduced upon heating, presumably due to desorption. Again, the relative difference of elemental composition of both foilswhich is important for the later investigation of the alloy influence, where the difference of the EMD's from both targets is taken-was in the $1 \%$ region.

SIMS analysis aimed at determining the sample composition as a function of distance from either the $\mathrm{Ni}$ or the $\mathrm{C}$ surface side of the foils. The depth profiling measurements ${ }^{40}$ on the free-standing foils were performed in a quadrupole based ion microprobe using a normally incident, raster scanned $2 \mathrm{keV} \mathrm{O}_{2}^{+}$primary ion beam for sputter erosion and secondary ion yield enhancement ${ }^{41}$ (scan width $140 \mu \mathrm{m}$, electronically gated area typically $40 \mu \mathrm{m}$ ). Raster scanning ion imaging ${ }^{42}$ in parallel with data acquisition during depth profiling provided qualitative information about the lateral uniformity in composition. Moreover, lateral differences in thickness could be assessed from the local differences in time required for complete removal of the sample ("breakthrough").

Figure 3(a) shows examples of depth profiles of $\mathrm{Ni}$ (dashed line) and $\mathrm{Cu}$ (solid line) measured from the Ni side of the sandwich. For ease of comparison, the profiles are presented in normalized form, ignoring surface effects. As the erosion rate could not be determined independently, the profiles are shown as a function of sputtering time. The $\mathrm{Ni}$ and $\mathrm{Cu}$ layers of the sandwich are clearly separated, but the interface is not sharp. This is attributed to the lateral nonuniformity of the carbon backing, presumably related to the roughness of the betaine spacer. The nonuniformity also gave rise to differences in breakthrough time by about $10 \%$. Within this margin, profiles measured at different spots on the sample were found to be reproducible. The effect of annealing on the sample composition is illustrated in Fig. 3(b). 


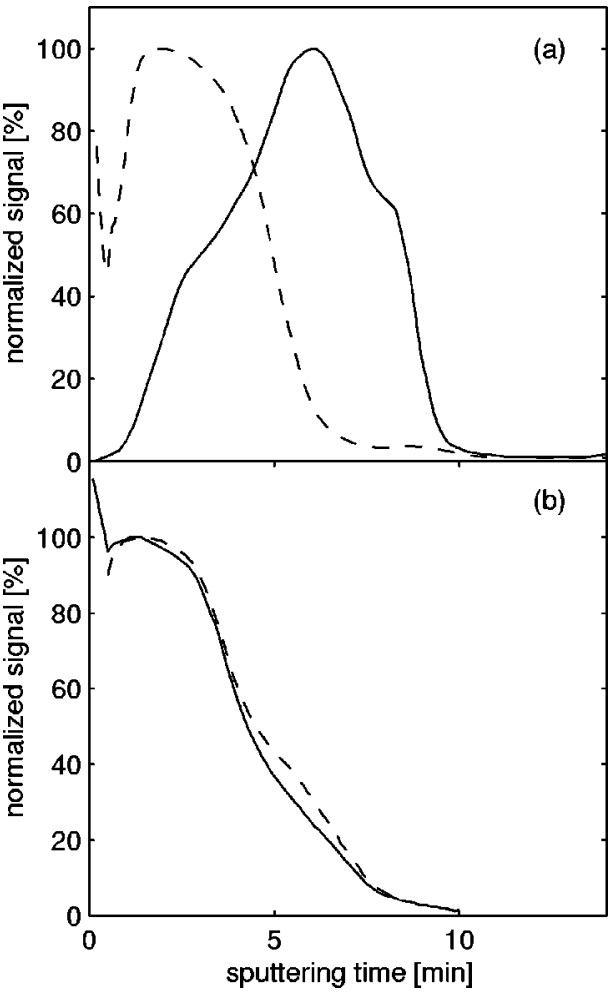

FIG. 3. $\mathrm{Cu}$ (solid line) and Ni (dashed line) concentration as a function of depth. (a) sandwich foil, (b) heated sandwich foil.

It is evident that, within experimental accuracy, the depth profiles of $\mathrm{Ni}$ (dashed line) and $\mathrm{Cu}$ (solid line) are identical. This result supports the idea that the heat treatment of the sandwich resulted in the formation of a uniform alloy. The same observation has been made by Röll and Reill ${ }^{43}$ for the interdiffusion of $\mathrm{Cu}-\mathrm{Ni}$ multilayers (the thickness of each layer was $45 \mathrm{~nm}$ ) after annealing at $450{ }^{\circ} \mathrm{C}$ for $30 \mathrm{~min}$ and by Suni, Nicolet, and Mäenpäa 44 for 150 -nm-thick Cu-Ni couples heated at $550^{\circ} \mathrm{C}$ for $15 \mathrm{~min}$. Identical distributions of $\mathrm{Ni}$ and $\mathrm{Cu}$ were also observed in depth profiling from the carbon side of the sample. The SIMS measurements revealed some contamination with $\mathrm{Fe}$, in accordance with Fig. 2. Furthermore, carbon was found to be present in the alloy at concentrations of about $3 \%$.

Some attention has been devoted to the problem of shortranged clustering in $\mathrm{Cu}_{x} \mathrm{Ni}_{1-x}$ alloys. Warren-Cowley shortrange-order parameters have been measured by diffuse neutron scattering. ${ }^{45,46}$ The measurements indicated that there is a fairly small tendency for $\mathrm{Cu}$ and $\mathrm{Ni}$ atoms in an alloy to prefer nearest neighbors of the same atomic species, but beyond the nearest-neighbor shell, the atomic arrangement is essentially random. Specifically, for $x=0.5$ the probability that the nearest neighbor of an atom is of the same species is 0.56 instead of 0.50 . This number holds for $550{ }^{\circ} \mathrm{C} .{ }^{45} \mathrm{At}$ first glance short-range clustering seems to contradict the absence of a miscibility gap since it is widely accepted that clustering will always precede or accompany spinodal decomposition. In fact, several authors have predicted a miscibility gap with a critical temperature below $450{ }^{\circ} \mathrm{C},{ }^{46-48}$ but since chemical diffusion constants are extremely low at these temperatures, atomic motion is essentially frozen out and prohibits thermodynamic equilibrium. We therefore conclude from the

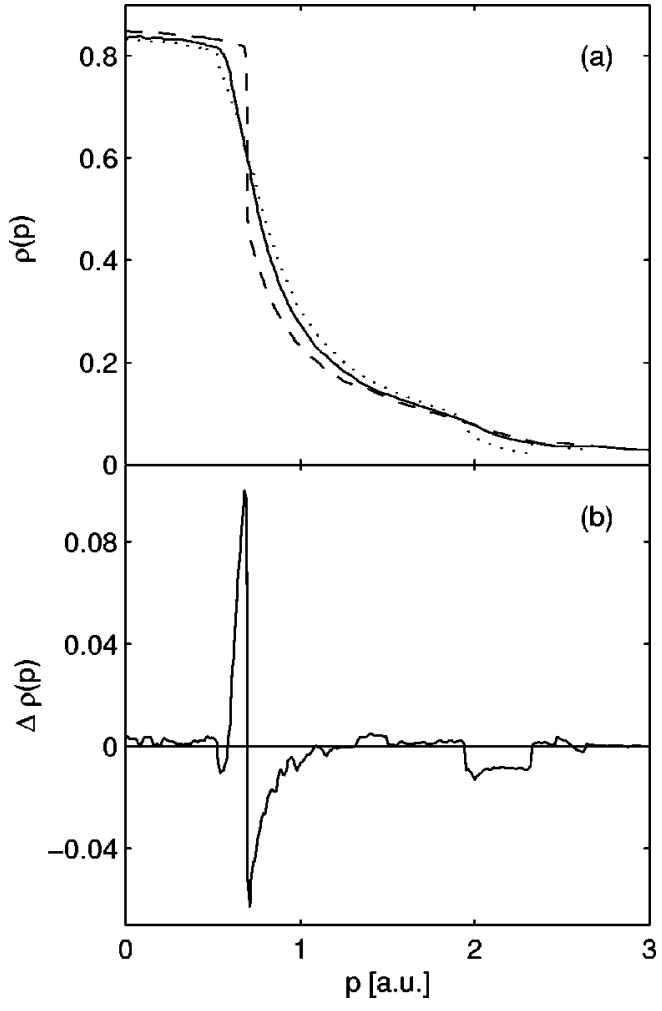

FIG. 4. The calculated EMD $\rho(p)$ of $\mathrm{Cu}$ (broken line), Ni (dotted line), and $\mathrm{Cu}_{0.50} \mathrm{Ni}_{0.50}$ (solid line) (a) and the difference $\Delta \rho$ as defined in Eq. (4.1) (b). From Ref. 19.

neutron-scattering experiments that the effect of clustering is small and can be neglected.

\section{EXPERIMENTAL RESULTS AND DISCUSSION}

Figure 4(a) shows the spherically averaged theoretical EMD's of $\mathrm{Cu}_{0.50} \mathrm{Ni}_{0.50}$ and the pure elements. ${ }^{19}$ Whereas the constituents have rather sharp discontinuities at the Fermi breaks - the radii of 0.76 a.u. for $\mathrm{Cu}$ and 0.60 a.u. for paramagnetic Ni correspond roughly to the bellies of the rather spherical FS of the sixth band for both cases ${ }^{49,50}$ - the break of the alloy is rounded off. It is well known that the eigenvalues $\varepsilon(\mathbf{p})$ of the band structure are real in a perfect crystal, but they become complex numbers in the alloy due to disorder-induced smearing of states. ${ }^{9}$ This is in contrast of the behavior of ordered alloys, which can be treated by more conventional band-structure calculations. ${ }^{51}$ The smearing implies that the alloy FS is not sharply defined but possesses a total width $2 \Delta p\left(\varepsilon_{F}\right)$, which amounts in the 50-50 alloy to 0.04 a.u. $^{52}$ It is also seen from Fig. 4(a) that the EMD's for $\mathrm{Ni}$ and the alloy are remarkably more intense than that for $\mathrm{Cu}$ for momenta above the FS, indicating the stronger influence of the more diffuse $d$ electrons in $\mathrm{Ni}$ and the alloy. Or, in other words, going from $\mathrm{Ni}$ to $\mathrm{Cu}$, the Fermi surface expands and becomes more pronounced, reflecting the increase of the $s-p$ character of the associated states. In Fig. 4(b) we have plotted the difference

$$
\Delta \rho=\left(\rho_{\mathrm{Cu}}+\rho_{\mathrm{Ni}}\right) / 2-\rho_{\mathrm{Cu}_{0.5} \mathrm{Ni}_{0.5}} .
$$

$\Delta \rho$ should be identical with the experimental accessible difference $\rho$ (sandwich) $-\rho$ (heated sandwich) and shows the 


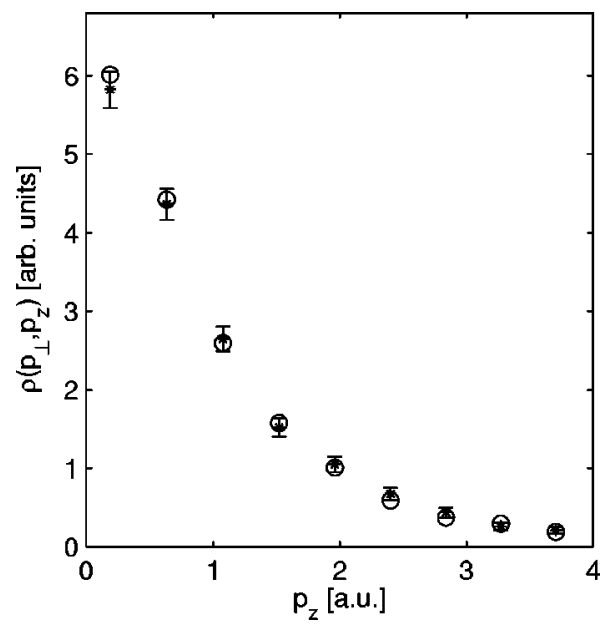

FIG. 5. The experimental EMD for the sandwich (stars) and the alloy (open circles) at $p_{\perp}=0.5$ as a function of $p_{z}$.

influence of alloying on the EMD. It is evident that the strongest effect is near the Fermi breaks, i.e., for momenta between 0.5 and 1 a.u.

In Fig. 5 both the EMD's of the sandwich (stars) and the alloy, i.e., the heated sandwich (open circles) at $p_{\perp}$ $=\sqrt{p_{x}^{2}+p_{y}^{2}}=0.5$ a.u., are plotted as a function of $p_{z}$. For this case differences should be observable near $p_{z}=0$, i.e., at large count rates. The experimental points of Fig. 5 have been normalized to the total number of coincidence events in each measurement, which are orders of magnitude larger than those which yield Fig. 5. It is readily seen that no difference within the error bars can be observed. Although our targets are isotropic, it is unfortunately not possible to increase statistics by adding up the coincidence events for a constant momentum $p$ since both the experimental resolutions in the three Cartesian directions of $\mathbf{p}$ are different (see Sec. III), and electron multiple-scattering influences the $p_{x}, p_{y}$ components stronger than $p_{z}$. But, nevertheless, to improve statistics, we have summed up all events for a constant $p_{z}$ to obtain what is called a coincident Compton profile $J_{\text {coinc }}$. Due to the limited range of our experiment in $p_{x}$ ( $\leqslant 1.6$ a.u.) and $p_{y}$ ( $\leqslant 2.5$ a.u.), $J_{\text {coinc }}$ is not identical with the noncoincident $\mathrm{CP}$. But in addition to the increase of the number of events, it has two other important advantages: Due to the limited $\left(p_{x}, p_{y}\right)$ range, the contribution of core states is strongly reduced and the trigger condition provides photon spectra free of any background radiation. $J_{\text {coinc }}$ has been evaluated in absolute units by normalizing it to the effective number $Z_{\text {eff }}$ of electrons that contribute to it. This number has been obtained by a Monte Carlo procedure that simulates the whole experiment including electron multiple scattering and the detailed geometry of both the photon and electron detector. The EMD's of Fig. 4(a) and that of graphite $^{53}$ have been used as input data. Due to the finite extension and the granularity of the electron detector, only a fraction $f_{i}$ of all recoil electrons, generated by photons scattered at the element $i=\mathrm{C}, \mathrm{Cu}$, or $\mathrm{Ni}$ and detected by the $\gamma$ detector, are measured. Having obtained $f_{i}$ from the $\mathrm{MC}$ calculation we have set

$$
Z_{\mathrm{eff}}=\sum_{i} f_{i} c_{i} Z_{i}
$$

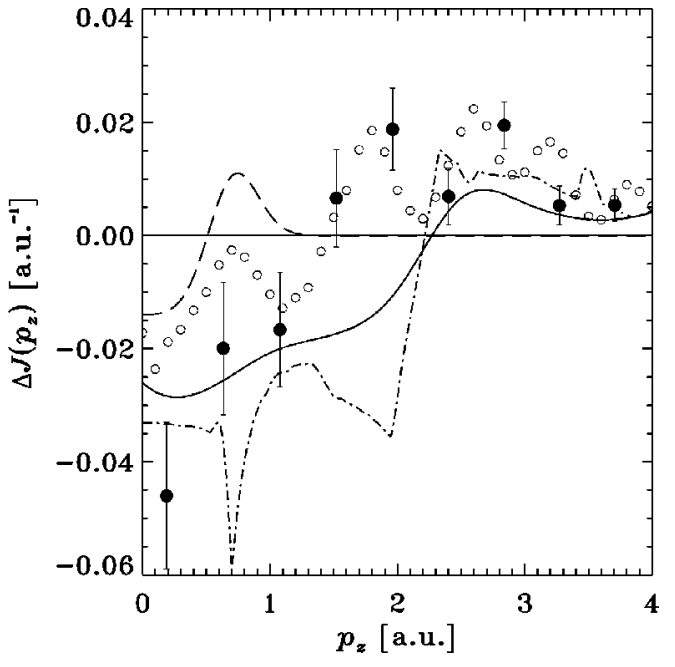

FIG. 6. The coincident $\mathrm{CP}$ difference $\Delta J$ (filled circles), the noncoincident difference (open circles), and $c\left(p_{z}\right) / 4$ from the KKRCPA calculation (solid line) (Ref. 19). For comparison the rigidband model (broken line) and the integration of the solid curve in Fig. 7 (dash-dotted line, not convoluted with experimental resolution) are also shown.

where $Z_{i}$ are the nuclear charges and $c_{i}$ are the relative atomic concentrations of the main elements that constitute the target foil. While electron multiple scattering has some influence on $Z_{\text {eff }}$, a change of the shape of $J_{\text {coinc }}$ due to this effect is negligible. This is also true if in the MC simulation the individual thicknesses of $\mathrm{C}, \mathrm{Cu}$, and $\mathrm{Ni}$ are increased by $50 \%$ (we know the absolute value of the thicknesses within $10 \%$ only). But in addition, only the difference of the coincident Compton profiles of the sandwich and the alloy will be discussed in the following, and therefore influences of multiple scattering should cancel, at least to first order.

Figure 6 shows the difference $\Delta J=J_{\text {coinc }}$ (sandwich)

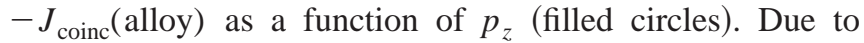
improved statistics, now an alloying effect is clearly observable. At $p_{z}=0$ it amounts to about $2 \%$ of the total coincident profile, i.e., it is a rather small effect. These findings are supported by $\mathrm{x}$-ray photoemission ${ }^{57}$ and $\mathrm{x}$-ray absorption spectroscopy, ${ }^{54}$ which show that the density of states of $\mathrm{Cu}-\mathrm{Ni}$ alloys can, to a very good approximation, be made up by superimposing those of $\mathrm{Ni}$ and $\mathrm{Cu}$. This means that there is indeed only a very limited sharing of electrons by the two constituents. Though the $\left(p_{x}, p_{y}\right)$ range is limited, it extends over the major part of the valence EMD. We therefore compare in Fig. 6 also with the experimental noncoincident $\mathrm{CP}$ difference ${ }^{19} \Delta J=\left(J_{\mathrm{Cu}}+J_{\mathrm{Ni}}\right) / 2-J_{\mathrm{Cu}_{0.5} \mathrm{Ni}_{0.5}}$. Despite the oscillations, the general trend of the experimental points (open circles) agrees nicely with our results. The oscillatory behavior might be an artifact introduced by the numerical procedure of data processing. ${ }^{19}$ Neither data processing like a deconvolution procedure accompanied by frequency filtering nor background subtraction has been applied to our data. This might be viewed in light of a remark made by Bansil et $a .^{20}$ that in their noncoincident Compton scattering experiments on $\mathrm{Cu}-\mathrm{Ni}$ single crystals absolute values of experimental CP's are difficult to interpret due to the presence of a background contribution of uncertain origin. The data of 


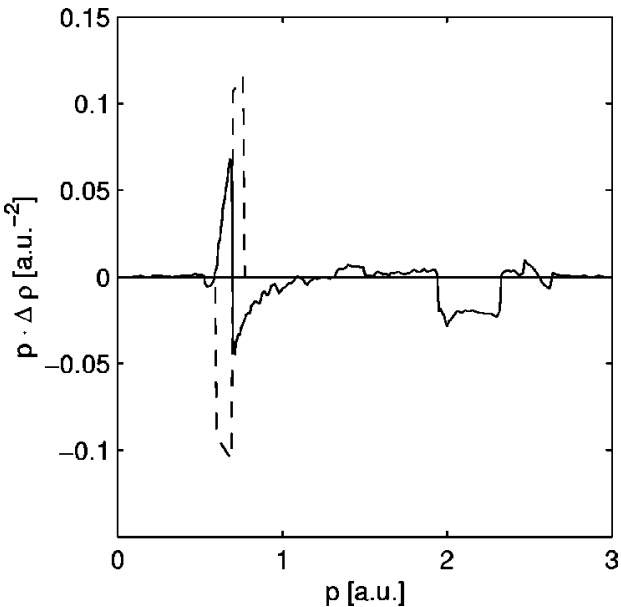

FIG. 7. $p \Delta \rho$ for the rigid-band model (broken line; multiplied by 0.5 ) and the KKR-CPA (solid line) as a function of momentum $p$

Benedek et al. ${ }^{19}$ for five $\mathrm{Cu}_{x} \mathrm{Ni}_{1-x}$ alloys and the two pure elements have been fitted by these authors to a common CP,

$$
J_{\mathrm{fit}}=a\left(p_{z}\right)+b\left(p_{z}\right)(x-0.5)+c\left(p_{z}\right)(x-0.5)^{2} .
$$

With our notation one obtains $\Delta J=c\left(p_{z}\right) / 4$. In the work of Benedek et al. ${ }^{19}$ also KKR-CPA theory has been parametrized in the form of Eq. (4.3). The solid line in Fig. 6 represents $c_{\text {theory }} / 4$. In view of the smallness of the effect, a very reasonable agreement between theory and experiment is observed.

For a more qualitative understanding of the alloy effect, we have also used a very simple rigid-band (RB) model, ${ }^{55}$ which has been discussed both in photoemission ${ }^{56-58}$ and Compton profile studies. ${ }^{19}$ Assuming that the Fermi momentum is given by $p_{F}=\alpha Z^{1 / 3}$ where $\alpha=\left(3 \pi^{2} / \Omega\right)^{1 / 3}(\Omega$ : atomic volume) remains essentially the same for the elements and the alloy, the only changing parameter is the valence $Z$. For the RB model one has

$$
Z_{\text {alloy }}=x Z_{\mathrm{Cu}}+(1-x) Z_{\mathrm{Ni}},
$$

which implies

$$
p_{F}^{3}(\text { alloy })=x p_{F}^{3}(\mathrm{Cu})+(1-x) p_{F}^{3}(\mathrm{Ni}) .
$$

Inserting the experimental belly radii of the pure constituents, one arrives for $x=0.5$ at $p_{F}$ (alloy) $=0.69$ a.u., a value which is identical with that found experimentally by the measurement of ferromagnetic exchange coupling across $\mathrm{Cu}_{x} \mathrm{Ni}_{1-x}$ layers. ${ }^{59,60}$ (Classical techniques like the de Haasvan Alphen effect or magnetoacoustic resonance are not applicable in concentrated alloys due to electron scattering introduced by alloy disorder. $\left.{ }^{61}\right)$ Starting with the EMD for free electrons $\rho(p)=\Omega /\left(4 \pi^{3}\right) \theta\left(p_{F}-p\right)$, where $\theta(p)$ is the step function, we have calculated the Compton profile difference $\Delta J$ and convoluted it with our $p_{z}$ resolution. The broken curve of Fig. 6 shows that this simple model is-at least for small momenta - in qualitative agreement with the experimental data. But it seems that this agreement is fortuitous. In Fig. 7 we have plotted the function $p \Delta \rho$ for both the RB model (broken curve; for better comparison we have multiplied by 0.5 ) and the EMD difference from KKR-CPA. It is evident that the intensity spikes at about 0.7 a.u. have a different sequence for both calculations. Integration of this function from a lower limit $p_{z}$ yields the Compton profile difference

$$
\Delta J\left(p_{z}\right)=2 \pi \int_{\left|p_{z}\right|}^{\infty} p \Delta \rho d p
$$

Whereas the Compton profile difference of the rigid-band model is determined by the EMD difference around 0.7 a.u., in KKR-CPA the corresponding contribution is small since the spikes at these momenta nearly cancel each other. In contrast, the KKR-CPA Compton profile difference $c\left(p_{z}\right) / 4$ in Fig. 6 is dominated by the negative feature around 2 a.u. (Fig. 7). This is demonstrated by the Compton profile difference $\Delta J$ obtained from the solid curve of Fig. 7 (dash-dotted curve in Fig. 6). It first reveals a reasonable agreement of the KKR-CPA Compton profile difference with the fitted curve $c\left(p_{z}\right) / 4$. Second, it demonstrates the compensation of the spikes by its strong extremum at about 0.7 a.u. If the lower integration limit $p_{z}$ has passed the first spike, the second one is no longer compensated yielding the strong minimum. Passing the second spike also, $\Delta J$ is nearly at the same value as before showing that the whole effect is dominated by the feature at about 2 a.u. in Fig. 7. It results from the behavior of the Fermi breaks near the $(1,1,2)$ reciprocal-lattice vector, which apparently survives the spherical averaging. Benedek et al. ${ }^{19}$ employed the special direction method ${ }^{62}$ and especially discussed its influence on the EMD near the Fermi breaks. In Ni rather strong $X$-centered hole ellipsoids in the fourth Brillouin zone dominate the Fermi surface, yielding a strong reduction of the EMD. On the contrary, for the 50-50 alloy and $\mathrm{Cu}$, the Fermi energy lies above the $d$ bands at this point, resulting in a less pronounced break. ${ }^{52,15}$ Altogether, this leads to a negative contribution of $\Delta \rho$ at about 2 a.u. Since this feature dominates $\Delta J$, it may happen that due to the limited range of our detector in $p_{x}$ direction our experimental points of Fig. 6 reflect a too small alloy effect. Nevertheless, we find it very remarkable that Umklapp contributions as discussed above are accessible to experimental observation.

\section{SUMMARY AND PERSPECTIVES}

We have measured the influence of alloying in a $\mathrm{Cu}_{0.50} \mathrm{Ni}_{0.50}$ alloy that has been produced by interdiffusion of 22-nm thin $\mathrm{Cu}$ and Ni films. Both the sandwich and the alloy have been characterized by XRF, ERDA, and SIMS. Coincident Compton profiles reveal a small difference between a simple mixing of the EMD's of the elements and that of the alloy. The effect can be explained by KKR-CPA calculations. Especially the use of ultrathin target foils opens the possibility to study a large class of random binary alloys that are produced far from thermodynamic equilibrium. Instead of producing the alloy by interdiffusion, they could be made either by simultaneous evaporation and condensation ${ }^{54}$ of the constituents or by cosputtering. In this way arbitrary alloy compositions are achieved that would otherwise be impossible to get from the melt. On the other hand, KKR-CPA 
calculations are independent from thermodynamic conditions. Cosputtered $\mathrm{Cu}_{x} \mathrm{Ni}_{1-x}$ films with $x$ between 0.12 and 0.87 have been studied by photoemission spectroscopy ${ }^{63}$ and found in better agreement with CPA calculations than earlier measurements ${ }^{56}$ due to improved resolution. This demonstrates that the production method yields reasonable results. At the same time it opens the possibility to study alloys where the size of the alloying effect is larger than in $\mathrm{Cu} / \mathrm{Ni}$ system, which was chosen due its complete miscibility.

\section{ACKNOWLEDGMENTS}

The authors thank Dr. W. Assmann from the Munich Heavy Ion Accelerator Group for the ERDA measurements. C.M. acknowledges a grant from the ESRF. This work was supported by the Bundesministerium für Bildung, Wissenschaft, Forschung und Technologie, Contracts Nos. 05 5WMAAI and 05 650HRA5.
*Present address: HASYLAB (DESY), Hamburg, Germany.

${ }^{1}$ M.J. Cooper, Rep. Prog. Phys. 48, 415 (1985).

${ }^{2}$ F. Bell, A.J. Rollason, J.R. Schneider, and W. Drube, Phys. Rev. B 41, 4887 (1990)

${ }^{3}$ F.F. Kurp, M. Vos, Th. Tschentscher, A.S. Kheifets, J.R. Schneider, E. Weigold, and F. Bell, Phys. Rev. B 55, 5440 (1997).

${ }^{4}$ Th. Tschentscher, J.R. Schneider, and F. Bell, Phys. Rev. B 48, 16965 (1993).

${ }^{5}$ M. Itou, S. Kishimoto, H. Kawata, M. Ozaki, H. Sakurai, and F. Itoh, J. Synchrotron Radiat. 5, 676 (1998); J. Phys. Soc. Jpn. 68, 515 (1999).

${ }^{6}$ R. Mayol and F. Salvat, At. Data Nucl. Data Tables 65, 55 (1997).

${ }^{7}$ W.B. Pearson, A Handbook of Lattice Spacings and Structures of Metals and Alloys (Pergamon, New York, 1958).

${ }^{8}$ A. Bansil, Z. Naturforsch., A: Phys. Sci. 48a, 165 (1993).

${ }^{9}$ A. Bansil, in Electronic Band Structure and its Applications, Lecture Note Series, Vol. 283, edited by M. Yussouff (Springer, Heidelberg, 1987), p. 273.

${ }^{10}$ J. Korringa, Physica (Utrecht) 13, 391 (1947).

${ }^{11}$ W. Kohn and N. Rostoker, Phys. Rev. 94, 1111 (1954).

${ }^{12}$ A. Bansil, L. Schwartz, and H. Ehrenreich, Phys. Rev. B 12, 2893 (1975).

${ }^{13}$ A. Bansil, Phys. Rev. Lett. 41, 1670 (1978).

${ }^{14}$ P.E. Mijnarends and A. Bansil, Phys. Rev. B 19, 2912 (1979).

${ }^{15}$ A. Bansil, Phys. Rev. B 20, 4035 (1979).

${ }^{16}$ A. Bansil, R.S. Rao, P.E. Mijnarends, and L. Schwartz, Phys. Rev. B 23, 3608 (1981).

${ }^{17}$ P.E. Mijnarends, Phys. Status Solidi A 102, 31 (1987).

${ }^{18}$ W.M. Temmerman, B.L. Gyorffy, and G.M. Stocks, J. Phys. F 8, 2461 (1978).

${ }^{19}$ R. Benedek, R. Prasad, S. Manninen, B.K. Sharma, A. Bansil, and P.E. Mijnarends, Phys. Rev. B 32, 7650 (1985).

${ }^{20}$ A. Bansil, S. Kaprzyk, A. Andrejczuk, L. Dobrzynski, J. Kwiatkowska, F. Maniawsky, and E. Zukowski, Phys. Rev. B 57, 314 (1998).

${ }^{21}$ M.J. Puska and R.M. Nieminen, Rev. Mod. Phys. 66, 841 (1994).

${ }^{22}$ B.W. Murray and J.D. McGervey, Phys. Rev. Lett. 24, 9 (1970).

${ }^{23}$ L.J. Rouse and P.G. Varlashkin, Phys. Rev. B 4, 2377 (1971).

${ }^{24}$ S. Tanigawa, S. Nanao, K. Kuribayashi, and M. Doyama, J. Phys. Soc. Jpn. 31, 1689 (1971).

${ }^{25}$ S. Nanao, K. Kuribayashi, S. Tanigawa, and M. Doyama, Phys. Lett. 38A, 489 (1972).

${ }^{26}$ M. Hasegawa, T. Suzuki, and M. Hirabayashi, J. Phys. Soc. Jpn. 37, 85 (1974).

${ }^{27}$ E. Bruno, B. Ginatempo, E.S. Giuliano, A.V. Ruban, and Yu.Kh. Vekilov, Phys. Rep. 249, 353 (1994).

${ }^{28}$ B.E.A. Gordon, W.E. Temmerman, and B.L. Gyorffy, J. Phys. F 11, 821 (1981).
${ }^{29}$ P. Suortti and Th. Tschentscher, Rev. Sci. Instrum. 66, 1798 (1995).

${ }^{30}$ A. Almazouzi, M.P. Macht, V. Naundorf, and G. Neumann, Phys. Rev. B 54, 857 (1996).

${ }^{31}$ J.M. Poate, K.N. Tu, and J.W. Mayer, Thin Films: Interdiffusion and Reactions (Wiley, New York, 1978).

${ }^{32}$ H. Lefakes, J.F. Cain, and P.S. Ho, Thin Solid Films 101, 207 (1983).

${ }^{33}$ R. Venos, W. Palmer, and H. Hoffmann, Thin Solid Films 162, 155 (1988).

${ }^{34}$ B.C. Johnson, C.L. Bauer, and A.G. Jordan, J. Appl. Phys. 59, 1147 (1986).

${ }^{35}$ A.D. LeClaire and G. Neumann, in Diffusion in Solid Metals and Alloys, edited by H. Mehrer, Landolt-Börnstein, New Series, Group III, Vol. 26, Chap. 3 (Springer, Heidelberg, 1990).

${ }^{36}$ H. Mehrer, in Diffusion in Solid Metals and Alloys (Ref. 35), Chap. 1.

${ }^{37}$ G. Brunel, G. Cizeron, and P. Lacombe, C. R. Seances Acad. Sci., Ser. B 269C, 895 (1969).

${ }^{38}$ D.B. Butrymowicz, J.R. Manning, and M.E. Read, in Diffusion Rate Data and Mass Transport Phenomena for Copper Systems, INCRA Series on the Metallurgy of Copper, Vol. 5 (International Copper Research Association, New York, 1977).

${ }^{39}$ W. Assmann, J.A. Davies, G. Dollinger, J.S. Forster, H. Huber, Th. Reichelt, and R. Siegele, Nucl. Instrum. Methods Phys. Res. B 118, 242 (1996).

${ }^{40} \mathrm{~K}$. Wittmaack, in Sputtering by Particle Bombardment III, Characteristics of Sputtered Particles, Technical Applications, Topics in Applied Physics Vol. 64, edited by R. Behrisch and K. Wittmaack (Springer, Berlin, 1991), Chap. 4.

${ }^{41}$ M.L. Yu and W. Reuter, J. Appl. Phys. 52, 1478 (1981).

${ }^{42}$ K. Wittmaack, Rev. Sci. Instrum. 47, 157 (1976).

${ }^{43}$ K. Röll and W. Reill, Thin Solid Films 89, 221 (1982).

${ }^{44}$ I. Suni, M.-A. Nicolet, and M. Mäenpää, Thin Solid Films 79, 69 (1981).

${ }^{45}$ B. Mozer, D.T. Keating, and S.C. Moss, Phys. Rev. 175, 868 (1968).

${ }^{46}$ J. Vrijen and S. Radelaar, Phys. Rev. B 17, 409 (1978).

${ }^{47}$ B. Predel, in Phase Equilibria, Crystallographic and Thermodynamic Data of Binary Alloys, edited by O. Madelung, LandoltBörnstein New Series, Group IV, Vol. 5d (Springer, Heidelberg, 1994), p. 203.

${ }^{48}$ L. Elford, F. Müller, and O. Kubaschewski, Ber. Bunsenges. Phys. Chem. 73, 601 (1969).

${ }^{49}$ G. Kontrym-Sznadj, H. Stachowiak, W. Wierzchowski, K. Petersen, N. Thrane, and G. Trumpy, J. Appl. Phys. 8, 151 (1975).

${ }^{50}$ A.P. Cracknell, in Electron States and Fermi Surfaces of Elements, edited by K.-H. Hellwege and J. L. Olsen, LandoltBörnstein New Series, Group III, Vol. 13c (Springer, Heidelberg, 1984). 
${ }^{51}$ S. Manninen, V. Honkimäki, K. Hämäläinen, J. Laukkanen, C. Blaas, J. Redinger, J. McCarthy, and P. Suortti, Phys. Rev. B 53, 7714 (1996).

${ }^{52}$ A. Bansil and P.E. Mijnarends, Phys. Rev. B 30, 628 (1984).

${ }^{53}$ C. Metz, Th. Tschentscher, P. Suortti, A.S. Kheifets, D.R. Lun, T. Sattler, J.R. Schneider, and F. Bell, J. Phys.: Condens. Matter 11, 3933 (1999).

${ }^{54}$ W. Gudat and C. Kunz, Phys. Status Solidi B 52, 433 (1972).

${ }^{55}$ N.F. Mott, Adv. Phys. 13, 325 (1964).

${ }^{56}$ D.H. Seib and W.E. Spicer, Phys. Rev. B 2, 1676 (1970); 2, 1694 (1970).

${ }^{57}$ S. Hüfner, G.K. Wertheim, R.L. Cohen, and J.H. Wernick, Phys.
Rev. Lett. 28, 488 (1972).

${ }^{58}$ H.H. Hsieh, Y.K. Chang, W.F. Pong, J.Y. Pieh, P.K. Tseng, T.K. Sham, F. Coulthard, S.J. Naftel, J.F. Lee, S.C. Chung, and K.L. Tsang, Phys. Rev. B 57, 15204 (1998).

${ }^{59}$ N.N. Lathiotakis, B.L. Gyorffy, and J.B. Staunton, J. Phys.: Condens. Matter 10, 10357 (1998).

${ }^{60}$ S.N. Okuno and K. Inomata, Phys. Rev. Lett. 70, 1711 (1993).

${ }^{61}$ I.M. Templeton and P.Z. Coleridge, J. Phys. F 5, 1307 (1975).

${ }^{62}$ R. Prasad and A. Bansil, Phys. Rev. B 21, 496 (1980).

${ }^{63}$ N.J. Shevchik and C.M. Penchina, Phys. Status Solidi B 70, 619 (1975). 